ESAIM: M2AN 48 (2014) 1227-1240

DOI: $10.1051 / \mathrm{m} 2 \mathrm{an} / 2013138$
ESAIM: Mathematical Modelling and Numerical Analysis

www.esaim-m2an.org

\title{
BASIC PRINCIPLES OF MIXED VIRTUAL ELEMENT METHODS
}

\author{
F. Brezzi ${ }^{1,2}$, Richard S. FAlK $^{3}$ And L. Donatella Marini ${ }^{4}$
}

\begin{abstract}
The aim of this paper is to give a simple, introductory presentation of the extension of the Virtual Element Method to the discretization of $H$ (div)-conforming vector fields (or, more generally, of $(n-1)-$ Cochains $)$. As we shall see, the methods presented here can be seen as extensions of the so-called BDM family to deal with more general element geometries (such as polygons with an almost arbitrary geometry). For the sake of simplicity, we limit ourselves to the 2-dimensional case, with the aim of making the basic philosophy clear. However, we consider an arbitrary degree of accuracy $k$ (the Virtual Element analogue of dealing with polynomials of arbitrary order in the Finite Element Framework).
\end{abstract}

Mathematics Subject Classification. 65N30, 65N12, 65N15, 76R50.

Received June 2, 2013. Revised October 27, 2013.

Published online July 15, 2014.

\section{INTRODUCTION}

The Virtual Element Method has been recently introduced as a generalization of $H^{1}$-conforming Finite Elements to arbitrary element-geometry and as a generalization of the 0 - Cochains version of Mimetic Finite Differences (see [13] or [8]) to arbitrary degree of accuracy and arbitrary continuity properties (see $[1,4,5,18]$ ).

The original nodal version of Virtual Elements put them, essentially, in the framework of non-polynomial Finite Elements and/or Finite Elements on polygonal (or polyhedral) meshes. For example, [23-25, 29-33].

The range of applicability of the basic ideas of Virtual Element Methods seems to be very wide, although a reliable knowledge of the types of real-life applications where these methods are to be recommended is, in our opinion, still lacking.

In this paper, we want to show the basic ideas of the possible extension of the Virtual Element framework to the discretization of $H$ (div)-conforming vector fields (as in Mixed Finite Element Methods). In doing so, we will generalize Mixed Finite Elements (see e.g., [11] or [14]) to arbitrary element geometry, and the original $(n-1)-$ Cochains version of Mimetic Finite Differences (see e.g., $[9,15-17,19,26-28]$; see also [10, 22] for overviews in more general frameworks) to arbitrary degree of accuracy.

Keywords and phrases. Mixed formulations, virtual elements, polygonal meshes, polyhedral meshes.

1 IUSS-Pavia and IMATI-CNR, Via Ferrata 1, 27100 Pavia, Italy. brezzi@imati.cnr.it

2 KAU, Jeddah, Saudi Arabia.

3 Department of Mathematics, Rutgers University, Piscataway, NJ 08854, USA. falk@math.rutgers.edu

4 Dipartimento di Matematica, Università di Pavia, and IMATI-CNR, Via Ferrata 1, 27100 Pavia, Italy. marini@imati.cnr.it 
For the sake of simplicity, we will just consider the basic case of 2-dimensional polygons and the simplest toy-problem of the mixed formulation of the Laplace operator (or, actually, operators of the form $\operatorname{div}(\mathbb{K}$ grad)), with the aim of making the general philosophy understood in the simplest possible case (as was done already in [4] for the $H^{1}$-conforming case).

Throughout the paper, for a bounded Lipschitz-continuous domain $\Omega$ and an integer $s$, we will use the common notation $(\cdot, \cdot)_{s, \Omega}$ and $\|\cdot\|_{s, \Omega}$ to denote the $H^{s}(\Omega)$ scalar product and norm, respectively. When no confusion can occur, the indication of the domain $\Omega$ will be omitted. Moreover, we shall often write $(\cdot, \cdot)$ and $\|\cdot\|$ to indicate the $L^{2}$ scalar product and norm, respectively. As usual, $|\cdot|_{s, \Omega}$ will denote the $H^{s}(\Omega)$ semi-norm (using only the derivatives of order $s$ ).

With an abuse of language, we shall often write "polynomial of degree $k$ " meaning actually "polynomial of degree $\leq k$ ". With another abuse of language, we will write the integral of the product of two functions even when the right notation would be a duality pairing. Finally, $C$ will denote a generic constant (independent of the mesh size), not necessarily the same from one occurrence to the other.

An outline of the paper is the following. In the next section, we shall present the continuous model problem and its mixed formulation. The decomposition and the corresponding discretizations will then be introduced in Section 3. The interpolation operators (from spaces of smooth functions to the discretized ones) will then be introduced in Section 4. In Section 5 we will present the discretized problem, and the proof of convergence and error estimates will be given in Section 6. At the end of Section 6, several remarks will give some hints on possible extensions of the method to more general situations.

Finally, we point out from the very beginning that "the name of the game" is that we know how to integrate "every polynomial on every polygon" through (for instance)

$$
\int_{E} x_{1}^{k}=\frac{1}{k+1} \int_{\partial E} x_{1}^{k+1} n_{1} \mathrm{~d} s
$$

and normal one-dimensional integration on the edges.

\section{The Continuous PRoBlem}

We consider the classical model problem of Darcy flow in a porous medium.

Given the (polygonal) computational domain $\Omega \subset \mathbb{R}^{2}$ and a source term $f \in L^{2}(\Omega)$, we then look for a scalar function $p \in H^{1}(\Omega)$ (pressure), such that

$$
-\operatorname{div}(\mathbb{K} \operatorname{grad} p)=f \quad \text { in } \Omega \quad(\mathbb{K} \operatorname{grad} p) \cdot \boldsymbol{n}=0 \text { on } \Gamma \equiv \partial \Omega,
$$

where the tensor $\mathbb{K}$ represents the permeability of the medium, and for simplicity we assumed no flux boundary conditions all over $\Gamma$. We assume that $\mathbb{K}$ is symmetric, positive definite and, for simplicity, constant (or piecewise constant).

We also denote by

$$
\|\mathbb{K}\| \quad \text { and } \quad\left\|\mathbb{K}^{-1}\right\|
$$

the matrix norm of $\mathbb{K}$ and $\mathbb{K}^{-1}$, respectively, in $\mathbb{R}^{2}$. Clearly, we must require that $f$ has zero mean value on $\Omega$, and $p$ will be determined only up to an additive constant. To fix ideas, we might choose the constant by requiring that $p$ have zero mean value as well. In other words, we have

$$
\int_{\Omega} f \mathrm{~d} \Omega=0 \quad \text { and } \quad \int_{\Omega} p \mathrm{~d} \Omega=0 .
$$

Introducing the variable $\boldsymbol{u}$ (often called "velocity") given by

$$
\boldsymbol{u}:=-\mathbb{K} \operatorname{grad} p
$$


the problem can be written in the so-called mixed form, that is

$$
\boldsymbol{u}=-\mathbb{K} \operatorname{grad} p, \quad \operatorname{div} \boldsymbol{u}=f \text { in } \Omega, \quad \boldsymbol{u} \cdot \boldsymbol{n}=0 \text { on } \Gamma .
$$

We consider the spaces

$$
H:=\left(L^{2}(\Omega)\right)^{2}, \quad V:=\{\boldsymbol{v} \in H(\operatorname{div} ; \Omega) \text { s.t. } \boldsymbol{v} \cdot \boldsymbol{n}=0 \text { on } \Gamma\}, \quad Q:=L^{2}(\Omega) / \mathbb{R},
$$

with norms

$$
\|\boldsymbol{v}\|_{H}^{2}=\int_{\Omega}|\boldsymbol{v}|^{2} \mathrm{~d} \Omega, \quad\|\boldsymbol{v}\|_{V}^{2}=\int_{\Omega}|\boldsymbol{v}|^{2} \mathrm{~d} \Omega+\int_{\Omega}|\operatorname{div} \boldsymbol{v}|^{2} \mathrm{~d} \Omega, \quad\|q\|_{Q}^{2}=\int_{\Omega}|q|^{2} \mathrm{~d} \Omega,
$$

and the bilinear forms

$$
\begin{aligned}
& a(\boldsymbol{u}, \boldsymbol{v}):=\int_{\Omega} \mathbb{K}^{-1} \boldsymbol{u} \cdot \boldsymbol{v} \mathrm{d} \Omega \quad \boldsymbol{u}, \boldsymbol{v} \in V \\
& b(\boldsymbol{v}, q):=\int_{\Omega} \operatorname{div} \boldsymbol{v} q \mathrm{~d} \Omega \quad \boldsymbol{v} \in V, q \in Q .
\end{aligned}
$$

Then we can write (2.5) in variational form: find $(\boldsymbol{u}, p) \in V \times Q$ such that:

$$
\begin{cases}a(\boldsymbol{u}, \boldsymbol{v})-b(\boldsymbol{v}, p)=0 & \forall \boldsymbol{v} \in V, \\ b(\boldsymbol{u}, q)=(f, q) & \forall q \in Q,\end{cases}
$$

where, in agreement with our notation,

$$
(f, q):=\int_{\Omega} f q \mathrm{~d} \Omega
$$

It is not difficult to check that

$$
\begin{gathered}
|a(\boldsymbol{u}, \boldsymbol{v})| \leq\|a\|\|\boldsymbol{u}\|_{H}\|\boldsymbol{v}\|_{H}, \quad \forall \boldsymbol{u}, \boldsymbol{v} \in H, \\
|b(\boldsymbol{u}, q)| \leq\|b\|\|\boldsymbol{u}\|_{V}\|q\|_{Q}, \quad \forall \boldsymbol{u} \in V, \forall q \in Q,
\end{gathered}
$$

where $\|a\|$ depends on $\mathbb{K}$ and $\|b\|$ is actually equal to 1 . We can also easily see that

$$
a(\boldsymbol{v}, \boldsymbol{v}) \geq \alpha\|\boldsymbol{v}\|_{H}^{2} \quad \forall \boldsymbol{v} \in H
$$

where $\alpha>0$ also depends on $\mathbb{K}$, and that there exists a $\beta>0$ such that

$$
\sup _{\boldsymbol{v} \in V} \frac{b(\boldsymbol{v}, q)}{\|\boldsymbol{v}\|_{V}} \geq \beta\|q\|_{Q} \quad \forall q \in Q,
$$

where $\beta$ depends on the domain $\Omega$. Introducing the kernel $\mathcal{K}$ as

$$
\mathcal{K}:=\{\boldsymbol{v} \in V \text { such that } b(\boldsymbol{v}, q)=0 \forall q \in Q\},
$$

we can finally see that

$$
\|\boldsymbol{v}\|_{H}=\|\boldsymbol{v}\|_{V} \quad \forall \boldsymbol{v} \in \mathcal{K} .
$$

Hence, we have that (2.10) has a unique solution, with

$$
\|\boldsymbol{u}\|_{V}+\|p\|_{Q} \leq C\|f\|_{0}
$$

where we recall that (here and in all the sequel), $C$ will denote a generic constant, non necessarily the same at all occurrences. 


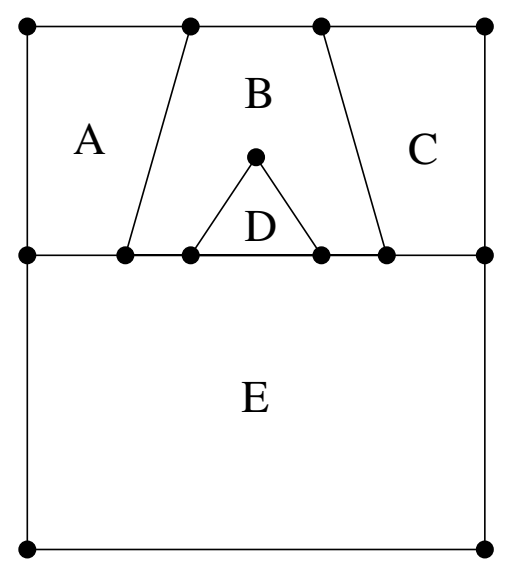

Figure 1. Elements $A$ and $C$ have 4 edges; $B$ has 7 edges; $D$ has 3 edges; $E$ has 8 edges.

\section{Decomposition And Discretization}

In order to approximate the solution of (2.10), we consider a sequence $\left\{\mathcal{T}_{h}\right\}_{h}$ of decompositions of $\Omega$ into sub-polygons, such that:

HD - There exists a positive real number $C_{\mathcal{T}}$ such that for every $h$ and for every $E \in \mathcal{T}_{h}$ :

- the ratio between the shortest edge and the diameter $h_{E}$ of $E$ is bigger than $C_{\mathcal{T}}$, and

- $E$ is star-shaped with respect to every point of a ball of radius $C_{\mathcal{T}} h_{E}$.

Remark 3.1. Assumptions HD have several important consequences that can be rather easily checked. First, every element will be simply connected. Moreover, there will exist an integer $N_{\mathcal{T}}$ (depending only on $C_{\mathcal{T}}$ ) such that the number of edges of every polygon $E$ in $\mathcal{T}$ is $\leq N_{\mathcal{T}}$. Finally, we observe that meshes traditionally considered as nonconforming, as the one in Figure 1, are easily allowed, although hanging nodes (or, here, hanging edges) will not be considered as slaves but (as we shall see) will carry, each, a set of independent unknowns.

Remark 3.2. If we assume that the permeability tensor $\mathbb{K}$ is piecewise constant (instead of globally constant), we will also assume that the decomposition is such that $\mathbb{K}$ is constant in every element.

Corresponding to the decomposition $\mathcal{T}_{h}$, we split the bilinear forms

$$
a(\boldsymbol{u}, \boldsymbol{v})=\sum_{E \in \mathcal{T}_{h}} a^{E}(\boldsymbol{u}, \boldsymbol{v}), \quad \boldsymbol{u}, \boldsymbol{v} \in H, \quad b(\boldsymbol{u}, q)=\sum_{E \in \mathcal{T}_{h}} b^{E}(\boldsymbol{u}, q), \quad \forall \boldsymbol{u} \in V, \forall q \in Q,
$$

and we consider, with obvious notation, the element by element norms

$$
\|\boldsymbol{v}\|_{H}^{2}=\sum_{E \in \mathcal{T}_{h}}\|\boldsymbol{v}\|_{0, E}^{2}, \quad\|\boldsymbol{v}\|_{V, h}^{2}=\sum_{E \in \mathcal{T}_{h}}\|\boldsymbol{v}\|_{V, E}^{2}, \quad\|q\|_{Q}^{2}=\sum_{E \in \mathcal{T}_{h}}\|q\|_{0, E}^{2}
$$

Clearly,

$$
\|\boldsymbol{v}\|_{V, h}^{2} \equiv\|\boldsymbol{v}\|_{V}^{2} \quad \text { whenever } \boldsymbol{v} \in V,
$$

while the spaces $Q$ and $H$ do not imply any continuity requirement. 
Then, for every integer $k \geq 1$, we consider the following finite dimensional spaces:

$V_{h}:=\left\{\boldsymbol{v} \in V\right.$ s.t. $(\boldsymbol{v} \cdot \boldsymbol{n})_{\mid e} \in \mathbb{P}_{k}(e)$ for all edge $e$ in $\mathcal{T}_{h}$,

$$
\begin{aligned}
& \left.(\operatorname{div} \boldsymbol{v})_{\mid E} \in \mathbb{P}_{k-1}(E) \text { and }(\operatorname{rot} \boldsymbol{v})_{\mid E} \in \mathbb{P}_{k-1}(E) \text { for all element } E \in \mathcal{T}_{h}\right\}, \\
Q_{h}:= & \left\{q \in Q \text { s.t. } q_{\mid E} \in \mathbb{P}_{k-1}(E) \text { for all element } E \in \mathcal{T}_{h}\right\} .
\end{aligned}
$$

Remark 3.3. Since every element is simply connected, it is elementary to check that a vector field $\boldsymbol{v}$ having $\boldsymbol{v} \cdot \boldsymbol{n}=0$ on $\partial E$, and both $\operatorname{div} \boldsymbol{v}=0$ and $\operatorname{rot} \boldsymbol{v}=0$ in $E$ is identically zero. This implies that the restriction of $V_{h}$ to each element is finite dimensional, the dimension being less than or equal to $k+1$ times the number of edges plus $k(k+1)$ (= twice the dimension of $\left.\mathbb{P}_{k-1}\right)$.

Remark 3.4. For every element $E$ in $\mathcal{T}_{h}$, it is also easy to check that every polynomial vector field of degree $\leq k$ (having normal component of degree $\leq k$ on each edge, divergence of degree $\leq k-1$ and rotational of degree $\leq k-1$ inside) will belong to the restriction of $V_{h}$ to $E$. Hence, $\left[\mathbb{P}_{k}(E)\right]^{2} \subset V_{h \mid E}$.

The choice of the degrees of freedom in $Q_{h}$ is trivial. On the other hand, in order to define the degrees of freedom in the space $V_{h}$, we define first the following notation: for $t$ a nonnegative integer and $e$ an edge with midpoint $x_{e}$ and length $h_{e}$, we denote by $\mathcal{M}_{t}^{e}$ the set of $t+1$ normalized monomials

$$
\mathcal{M}_{t}^{e}:=\left\{1, \frac{x-x_{e}}{h_{e}},\left(\frac{x-x_{e}}{h_{e}}\right)^{2}, \ldots,\left(\frac{x-x_{e}}{h_{e}}\right)^{t}\right\} .
$$

Similarly, for a two-dimensional domain $E$ with diameter $h_{E}$ and barycenter $\boldsymbol{x}_{E}$, we denote by $\mathcal{M}_{t}^{E}$ the set of $(t+1)(t+2) / 2$ normalized monomials

$$
\mathcal{M}_{t}^{E}:=\left\{\left(\frac{x-\boldsymbol{x}_{E}}{h_{E}}\right)^{\boldsymbol{\beta}}, \quad|\boldsymbol{\beta}| \leq t\right\},
$$

where, as usual, for a nonnegative multi-index $\boldsymbol{\beta}=\left(\beta_{1}, \beta_{2}\right)$, we set $|\boldsymbol{\beta}|=\beta_{1}+\beta_{2}$ and $\boldsymbol{x}^{\boldsymbol{\beta}}=x_{1}^{\beta_{1}} x_{2}^{\beta_{2}}$. We also assume that, in particular, for all $E \in \mathcal{T}_{h}$ and for all nonnegative integer $t$, the elements $q^{i} \in \mathcal{M}_{t}^{E}$ are ordered in such a way that $q^{1}$ is the constant 1 in $E$.

In $V_{h}$ we define then the following degrees of freedom:

$$
\begin{aligned}
& \text { 1. } \int_{e} \boldsymbol{v} \cdot \boldsymbol{n} q \mathrm{~d} t \quad \forall q \in \mathcal{M}_{k}^{e} \quad \forall \text { edge } e \text { in } \mathcal{T}_{h}, \\
& \text { 2. } \int_{E} \boldsymbol{v} \cdot \operatorname{grad} q \mathrm{~d} \Omega \quad \forall q \in \mathcal{M}_{k-1}^{E} \backslash\{1\} \quad \forall E \in \mathcal{T}_{h}, \\
& \text { 3. } \int_{E} \operatorname{rot} \boldsymbol{v} q \mathrm{~d} \Omega \quad \forall q \in \mathcal{M}_{k-1}^{E} \quad \forall E \in \mathcal{T}_{h} .
\end{aligned}
$$

To analyze the unisolvence, it will be more convenient to proceed on an element by element basis.

Proposition 3.5. Let $E$ be an element in $\mathcal{T}_{h}$, let $\ell$ be the number of its edges, and let $k$ be an integer $\geq 1$. Set

$$
n_{k}^{E}:=\{\ell(k+1)\}+\{k(k+1) / 2-1\}+\{k(k+1) / 2\} \equiv \ell(k+1)+k^{2}+k-1
$$

and define the local space

$V_{h}^{E}:=\{\boldsymbol{v} \in H(\operatorname{div} ; E) \cap H(\operatorname{rot} ; E)$ such that

$$
\left.(\boldsymbol{v} \cdot \boldsymbol{n})_{\mid e} \in \mathbb{P}_{k}(e) \text { for all edge e of } E,(\operatorname{div} \boldsymbol{v})_{\mid E} \in \mathbb{P}_{k-1}(E) \text { and }(\operatorname{rot} \boldsymbol{v})_{\mid E} \in \mathbb{P}_{k-1}(E)\right\} \text {. }
$$

Then the $n_{k}^{E}$ degrees of freedom (3.8) (restricted to $E$ ) are unisolvent in $V_{h}^{E}$. 
Proof. We remark first that the degrees of freedom (3.8) restricted to $E$ determine, in a unique way,

$$
\begin{aligned}
& \text { 1. } \int_{e} \boldsymbol{v} \cdot \boldsymbol{n} q \mathrm{~d} t \quad \forall q \in \mathbb{P}_{k}(e) \quad \forall \text { edge } e \text { of } E \\
& \text { 2. } \int_{E} \boldsymbol{v} \cdot \operatorname{grad} q \mathrm{~d} \Omega \quad \forall q \in \mathbb{P}_{k-1}(E) \backslash\{1\}, \\
& \text { 3. } \int_{E} \operatorname{rot} \boldsymbol{v} q \mathrm{~d} \Omega \quad \forall q \in \mathbb{P}_{k-1}(E) .
\end{aligned}
$$

In turn, the degrees of freedom (3.11) determine in a unique way,

- a function $g$ on $\partial E$, polynomial of degree $k$ on each edge, such that

$$
\int_{e} g q \mathrm{~d} s=\int_{e} \boldsymbol{v} \cdot \boldsymbol{n} q \mathrm{~d} s \quad \forall q \in \mathbb{P}_{k}(e) \quad \forall \text { edge } e \text { of } \partial E ;
$$

- a polynomial $r$ of degree $k-1$ in $E$ such that

$$
\int_{E} r q \mathrm{~d} \Omega=\int_{E} \operatorname{rot} \boldsymbol{v} q \mathrm{~d} \Omega \quad \forall q \in \mathbb{P}_{k-1}(E)
$$

- a polynomial $d$ of degree $k-1$ in $E$ such that

$$
\int_{E} d q \mathrm{~d} \Omega=-\int_{E} \boldsymbol{v} \cdot \operatorname{grad} q \mathrm{~d} \Omega+\int_{\partial E} \boldsymbol{v} \cdot \boldsymbol{n} q \mathrm{~d} s \quad \forall q \in \mathbb{P}_{k-1}(E) .
$$

Clearly $g, r$, and $d$ are the candidates to be, respectively, $\boldsymbol{v} \cdot \boldsymbol{n}$ on $\partial E$, and $\operatorname{rot} \boldsymbol{v}$ and $\operatorname{div} \boldsymbol{v}$ in $E$. This is obvious for $g$ and $r$, (from (3.11) (1) and (3) respectively), and is reasonably clear also for $d$, since

$$
\int_{E} \operatorname{div} \boldsymbol{v} q \mathrm{~d} \Omega=-\int_{E} \boldsymbol{v} \cdot \operatorname{grad} q \mathrm{~d} \Omega+\int_{\partial E} \boldsymbol{v} \cdot \boldsymbol{n} q \mathrm{~d} s \quad \forall q \in \mathbb{P}_{k-1}
$$

Then we consider two auxiliary problems, looking for functions $\psi$ and $\varphi$ in $H^{1}(E)$ such that:

$$
\Delta \psi=r \quad \text { in } E, \quad \psi=0 \quad \text { on } \partial E,
$$

and

$$
\Delta \varphi=d \quad \text { in } E, \quad \frac{\partial \varphi}{\partial n}=g \quad \text { on } \partial E,
$$

where we took into account that taking $q=1$ in (3.14) (and remembering that (3.12) gives $g=\boldsymbol{v} \cdot \boldsymbol{n}$ on $\partial \Omega$ ) we have

$$
\int_{E} d \mathrm{~d} \Omega=\int_{\partial E} g \mathrm{~d} s
$$

so that problem (3.17) has a solution. Setting $\boldsymbol{v}:=\operatorname{grad} \varphi-\operatorname{rot} \psi$, we immediately have

$$
\operatorname{div} \boldsymbol{v}=d \quad \text { in } E, \quad \operatorname{rot} \boldsymbol{v}=r \quad \text { in } E, \text { and } \quad \boldsymbol{v} \cdot \boldsymbol{n}=g \quad \text { on } \partial E .
$$

Since the domain $E$ is simply connected, we easily deduce that the function $\boldsymbol{v}$ satisfying (3.19) is unique and the proof is concluded. 
Remark 3.6. We note that, if the element $E$ is a triangle, and if, for instance, $k=1$, then the local space $V_{h}^{E}$, as defined in (3.10), has dimension equal to 7 , which is in between the dimension of the lowest order BDM element (i.e., $B D M_{1}(E)$, which coincides with $\left(\mathbb{P}_{1}(E)\right)^{2}$ and has dimension 6) and that of the "next to the lowest" Raviart-Thomas element (i.e., $R T_{1}(E)$, which is given by $\left(\mathbb{P}_{1}\right)^{2}+\mathbf{x} \mathbb{P}_{1}$ and has dimension 8). Indeed, in this case $V_{h}^{E}$ can be obtained by adding to $\left(\mathbb{P}_{1}(E)\right)^{2}$ the vector $\boldsymbol{v}=\operatorname{rot} \psi$, with $\psi$ satisfying

$$
\Delta \psi=1 \text { in } E \text { with } \psi=0 \text { on } \partial E \text {. }
$$

Note that if $E$ is an equilateral triangle, then the function $\psi$ in (3.20) coincides with the cubic bubble $b_{3}^{E}$, and then one has

$$
B D M_{1}(E) \subset V_{h}^{E} \subset B D M_{2}^{R}(E),
$$

where $B D M_{2}^{R}(E)$ is the so called "reduced BDM" space, made of those vectors in $B D M_{2}\left(\equiv\left(\mathbb{P}_{2}\right)^{2}\right)$ whose normal component has degree $\leq 1$ on each edge. It is immediate to see that $B D M_{2}^{R}(E)$ has dimension 9 , and (as is well known) can be obtained (for a general triangle) by adding to $R T_{1}(E)$ the vector $\operatorname{rot} b_{3}^{E}$. Instead, as we have seen, on the equilateral triangle, adding $\operatorname{rot} b_{3}$ to $B D M_{1}(E)$, we obtain our Virtual Element space $V_{h}(E)$. However, if $E$ is not equilateral, the inclusion $B D M_{1}(E) \subset V_{h}^{E}$ still holds, but $V_{h}^{E}$ will not be a subset of $B D M_{2}^{R}(E)$, as, in general, the function $\psi$ given by (3.20) is not a polynomial.

\section{INTERPOLATION IN $V_{h}$ AND $Q_{h}$}

The space $Q_{h}$ has a particularly simple structure. Hence, for a given $p \in Q$, we can immediately define its "interpolant" $p_{I} \in Q_{h}$ by

$$
\int_{E}\left(p-p_{I}\right) q_{k-1} \mathrm{~d} \Omega=0 \quad \forall E \in \mathcal{T}_{h}, \forall q_{k-1} \in \mathbb{P}_{k-1}(E) .
$$

Taking $q_{k-1} \equiv 1$ in each $E$ and summing over the elements, we easily get

$$
\int_{\Omega}\left(p-p_{I}\right) \mathrm{d} \Omega=\sum_{E \in \mathcal{T}_{h}} \int_{E}\left(p-p_{I}\right) \mathrm{d} \Omega=0,
$$

so that when $p \in Q$ (recall: with zero mean value over $\Omega$ ), we have that $p_{I} \in Q$ as well.

Note that we could write, locally, $p_{I}=P_{k-1}^{E} p$, where $P_{k-1}^{E}$ is the $L^{2}$-projection operator on $\mathbb{P}_{k-1}(E)$, and globally $p_{I}=P_{Q_{h}} p$ where $P_{Q_{h}}$ is the $L^{2}$-projection on $Q_{h}$.

As a consequence, we easily have (see e.g., [12]) that for every $h$, for every $E \in \mathcal{T}_{h}$, and for every integer $r$ with $0 \leq r \leq k$ :

$$
\left\|p-p_{I}\right\|_{0, E} \leq C h_{E}^{r}|p|_{r, E},
$$

where $C$ depends only on the constant $C_{\mathcal{T}}$ in $\mathbf{H D}$.

The interpolation in the space $V_{h}$ is also reasonably easy. We consider, for simplicity, a $\boldsymbol{w} \in V$, and we require additionally that for each element $E$ we have $\boldsymbol{w} \in \cap\left(L^{s}(\Omega)\right)^{2}$ for some $s>2$ (so that we can easily take its traces on each individual edge), and also $\operatorname{rot} \boldsymbol{w} \in L^{1}(E)$ (so that we can take its moments in $E$ ). Then we define its interpolant $\boldsymbol{w}_{I} \in V_{h}$ by requiring that

$$
\left\{\begin{array}{lll}
\int_{e}\left(\boldsymbol{w}-\boldsymbol{w}_{I}\right) \cdot \boldsymbol{n} q \mathrm{~d} t=0 & \forall q \in \mathcal{M}_{k}^{e} & \forall \text { edge } e \text { in } \mathcal{T}_{h}, \\
\int_{E}\left(\boldsymbol{w}-\boldsymbol{w}_{I}\right) \cdot \operatorname{grad} q \mathrm{~d} \Omega=0 & \forall q \in \mathcal{M}_{k-1}^{E} & \forall E \in \mathcal{T}_{h}, \\
\int_{E} \operatorname{rot}\left(\boldsymbol{w}-\boldsymbol{w}_{I}\right) q \mathrm{~d} \Omega=0 & \forall q \in \mathcal{M}_{k-1}^{E} \quad \forall E \in \mathcal{T}_{h} .
\end{array}\right.
$$


Using Proposition 3.5, it is not difficult to check that such a $\boldsymbol{w}_{I}$ exists and is unique in $V_{h}$. It is also easy to see that for every element $E$ and for every $q_{k-1} \in \mathbb{P}_{k-1}(E)$, we have

$$
\int_{E} \operatorname{div}\left(\boldsymbol{w}-\boldsymbol{w}_{I}\right) q_{k-1} \mathrm{~d} \Omega=-\int_{E}\left(\boldsymbol{w}-\boldsymbol{w}_{I}\right) \cdot \operatorname{grad} q_{k-1} \mathrm{~d} \Omega+\int_{\partial E}\left(\boldsymbol{w}-\boldsymbol{w}_{I}\right) \cdot \boldsymbol{n} q_{k-1} \mathrm{~d} s=0
$$

and since $\operatorname{div} \boldsymbol{w}_{I}$ itself is in $\mathbb{P}_{k-1}(E)$, we deduce that in each $E$,

$$
\operatorname{div} \boldsymbol{w}_{I}=P_{k-1}^{E} \operatorname{div} \boldsymbol{w}
$$

implying, as in (4.3), that

$$
\left\|\operatorname{div} \boldsymbol{w}-\operatorname{div} \boldsymbol{w}_{I}\right\|_{0, E} \leq C h_{E}^{r}|\operatorname{div} \boldsymbol{w}|_{r, E} \quad 0 \leq r \leq k .
$$

Moreover, using again [12] and classical arguments, we have also, for each $h$, for each $E \in \mathcal{T}_{h}$, and for each integer $r$ with $1 \leq r \leq k+1$,

$$
\left\|\boldsymbol{w}-\boldsymbol{w}_{I}\right\|_{0, E} \leq C h_{E}^{r}|\boldsymbol{w}|_{r, E}
$$

for some constant $C$ depending only on the constant $C_{\mathcal{T}}$ in HD.

Remark 4.1. The use of spaces of vector valued functions in $\left(L^{s}(E)\right)^{2} \cap H$ (div; $E$ ) for some $s>2$ in order to define the trace of the normal component on a single edge goes back to the early days of mixed methods (see e.g., [14]). Among the more recent and detailed use of this tool, we refer, for instance, to [15] (Lem. 4.1) for a detailed description of the reason why $s>2$ is sufficient, and to [11] (Rem. 2.5.1) for a detailed description of the reason why $s=2$ is not sufficient. On the other hand, in order to take the "integral" of rot $\boldsymbol{w}$ times a smooth function, we could just require $\operatorname{rot} \boldsymbol{w} \in H^{-1 / 2}(E)$.

\section{THE DISCRETE BILINEAR FORMS}

For every decomposition $\mathcal{T}_{h}$ and for every positive integer $k$, we have defined in (3.4) and (3.5) the spaces $V_{h}$ and $Q_{h}$, respectively. Now we need to define, on them, a discrete version of the bilinear forms $a$ and $b$ given in $(2.8)-(2.9)$.

For the bilinear form $b$, this is really simple. Indeed we can keep

$$
b(\boldsymbol{v}, q):=\int_{\Omega} \operatorname{div} \boldsymbol{v} q \mathrm{~d} \Omega \equiv \sum_{E \in \mathcal{T}_{h}} \int_{E} \operatorname{div} \boldsymbol{v} q \mathrm{~d} \Omega \quad \boldsymbol{v} \in V_{h}, q \in Q_{h},
$$

as in (2.9), which is easily computable since both $\operatorname{div} \boldsymbol{v}$ and $q$ are polynomials in each element. We explicitly point out that, as can be seen from (3.14) and (3.19), the divergence of any vector $\boldsymbol{v}$ in $V_{h}^{E}$ can be easily computed from knowledge of the degrees of freedom of $\boldsymbol{v}$.

On the other hand, the bilinear form $a$ needs to be dealt with in a more careful way. We start by noting that, on each element $E$, whenever $\widehat{\boldsymbol{u}}$ is of the form

$$
\widehat{\boldsymbol{u}}=\mathbb{K} \operatorname{grad} \widehat{q}_{k+1} \quad \text { with } \widehat{q}_{k+1} \in \mathbb{P}_{k+1},
$$

then for every $\boldsymbol{v} \in V_{h}^{E}$, we have

$$
\begin{aligned}
a^{E}(\widehat{\boldsymbol{u}}, \boldsymbol{v}) & =\int_{E} \mathbb{K}^{-1} \widehat{\boldsymbol{u}} \cdot \boldsymbol{v} \mathrm{d} \Omega=\int_{E} \operatorname{grad} \widehat{q}_{k+1} \cdot \boldsymbol{v} \mathrm{d} \Omega \\
& =-\int_{E} \widehat{q}_{k+1} \operatorname{div} \boldsymbol{v} \mathrm{d} \Omega+\int_{\partial E} \widehat{q}_{k+1} \boldsymbol{v} \cdot \boldsymbol{n} \mathrm{d} s .
\end{aligned}
$$


Since both $\operatorname{div} \boldsymbol{v}$ and $\boldsymbol{v} \cdot \boldsymbol{n}_{\mid \partial E}$ are easily computable from the degrees of freedom of $\boldsymbol{v}$ (as pointed out in (3.12) and in (3.14)), we have that (5.3) is also computable using only the degrees of freedom of $\boldsymbol{v}$. Inspired by this observation (that in turn is inspired by $[7,9]$ ), we define first, for each element $E$, the space

$$
\widehat{V}^{E}:=\left\{\widehat{\boldsymbol{v}} \in V_{h}^{E} \text { such that } \widehat{\boldsymbol{v}}=\mathbb{K} \operatorname{grad} \widehat{q}_{k+1} \text { for some } \widehat{q}_{k+1} \in \mathbb{P}_{k+1}(E)\right\} .
$$

Then we define the (oblique) projector $\widehat{\Pi}^{E}$ from $H(\operatorname{div} ; E)$ onto $\widehat{V}^{E}$ by

$$
a^{E}\left(\boldsymbol{v}-\widehat{\Pi}^{E} \boldsymbol{v}, \widehat{\boldsymbol{w}}\right)=0 \quad \forall \widehat{\boldsymbol{w}} \in \widehat{V}^{E},
$$

and we point out that $\widehat{\Pi}^{E} \boldsymbol{v}$ is explicitly computable for every $\boldsymbol{v} \in V_{h}^{E}$ using only its degrees of freedom. At this point, we consider the bilinear form $\mathcal{S}^{E}$, defined on $V_{h}^{E} \times V_{h}^{E}$, associated to the identity matrix in $\mathbb{R}_{k}^{n_{k}^{E}}$ with respect to the local basis determined by the degrees of freedom (3.8), and we define, in each $E$,

$$
a_{h}^{E}(\boldsymbol{u}, \boldsymbol{v}):=a^{E}(\widehat{\Pi} \boldsymbol{u}, \widehat{\Pi} \boldsymbol{v})+\left\|\mathbb{K}^{-1}\right\| \mathcal{S}^{E}((I-\widehat{\Pi}) \boldsymbol{u},(I-\widehat{\Pi}) \boldsymbol{v}) \quad \forall \boldsymbol{u}, \boldsymbol{v} \in V_{h}^{E}
$$

and, in a natural way,

$$
a_{h}(\boldsymbol{u}, \boldsymbol{v}):=\sum_{E \in \mathcal{T}_{h}} a_{h}^{E}(\boldsymbol{u}, \boldsymbol{v}) \quad \forall \boldsymbol{u}, \boldsymbol{v} \in V_{h} .
$$

Remark 5.1. It is important to observe that each of the degrees of freedom in (3.8) scales like $\boldsymbol{v}$ times $h_{E}$ : indeed, the choice of the monomials (3.6) and (3.7) was done exactly for this purpose. As a consequence, for instance, the sum of their squares (that, in the computer, will be the square of the usual Euclidean norm) will scale like $\|\boldsymbol{v}\|_{0, E}^{2}$. As a consequence, on every element $E$, the bilinear form $\mathcal{S}^{E}(\boldsymbol{v}, \boldsymbol{v})$ will scale as $a^{E}(\boldsymbol{v}, \boldsymbol{v})$. In particular we will have that there exist two positive constants, $c_{0}$ and $c_{1}$, depending only on $C_{\mathcal{T}}$ and $\mathbb{K}$ such that

$$
c_{0} a^{E}(\boldsymbol{v}, \boldsymbol{v}) \leq\left\|\mathbb{K}^{-1}\right\| \mathcal{S}^{E}(\boldsymbol{v}, \boldsymbol{v}) \leq c_{1} a^{E}(\boldsymbol{v}, \boldsymbol{v}) \forall E \in \mathcal{T}_{h}, \forall \boldsymbol{v} \in V^{E} .
$$

Proposition 5.2. The bilinear form $a_{h}$ defined in (5.6) has the following two properties:

$$
\begin{gathered}
a_{h}^{E}(\widehat{\boldsymbol{u}}, \boldsymbol{v})=a^{E}(\widehat{\boldsymbol{u}}, \boldsymbol{v}) \quad \forall E \in \mathcal{T}_{h}, \forall \widehat{\boldsymbol{u}} \in \widehat{V}^{E}, \forall \boldsymbol{v} \in V_{h}^{E}, \\
\exists \alpha^{*}, \alpha_{*}>0 \text { s.t. } \alpha_{*} a^{E}(\boldsymbol{v}, \boldsymbol{v}) \leq a_{h}^{E}(\boldsymbol{v}, \boldsymbol{v}) \leq \alpha^{*} a^{E}(\boldsymbol{v}, \boldsymbol{v}) \quad \forall E \in \mathcal{T}_{h}, \forall \boldsymbol{v} \in V_{h}^{E} .
\end{gathered}
$$

Proof. Property (5.9) follows immediately from (5.5) and (5.6): indeed for $\widehat{\boldsymbol{u}} \in \widehat{V}^{E},(5.5)$ implies that $S^{E}(\widehat{\boldsymbol{u}}-$ $\left.\widehat{\Pi}^{E} \widehat{\boldsymbol{u}}, \boldsymbol{v}-\widehat{\Pi}^{E} \boldsymbol{v}\right)=0 \forall \boldsymbol{v}$, and hence

$$
a_{h}^{E}(\widehat{\boldsymbol{u}}, \boldsymbol{v})=a^{E}\left(\widehat{\Pi}^{E} \widehat{\boldsymbol{u}}, \widehat{\Pi}^{E} \boldsymbol{v}\right)=a^{E}\left(\widehat{\Pi}^{E} \widehat{\boldsymbol{u}}, \boldsymbol{v}\right)=a^{E}(\widehat{\boldsymbol{u}}, \boldsymbol{v}) .
$$

Moreover, from (5.5) we have $a^{E}\left(\boldsymbol{v}-\widehat{\Pi}^{E} \boldsymbol{v}, \widehat{\boldsymbol{w}}\right)=0$ for all $\widehat{\boldsymbol{w}} \in \widehat{V}^{E}$ and for all $\boldsymbol{v} \in V_{h}^{E}$, so that by the Pythagorean theorem, we have

$$
a^{E}\left(\boldsymbol{v}-\widehat{\Pi}^{E} \boldsymbol{v}, \boldsymbol{v}-\widehat{\Pi}^{E} \boldsymbol{v}\right)+a^{E}\left(\widehat{\Pi}^{E} \boldsymbol{v}, \widehat{\Pi}^{E} \boldsymbol{v}\right)=a^{E}(\boldsymbol{v}, \boldsymbol{v}) \quad \forall \boldsymbol{v} \in V_{h}^{E} .
$$

Property (5.10) then follows easily using (5.6), (5.8), and then (5.12). Indeed, taking $\alpha^{*}$ as $\alpha^{*}:=\max \left\{1, c_{1}\right\}$, we have

$$
\begin{aligned}
a_{h}^{E}(\boldsymbol{v}, \boldsymbol{v}) & \leq a^{E}\left(\widehat{\Pi}^{E} \boldsymbol{v}, \widehat{\Pi}^{E} \boldsymbol{v}\right)+c_{1} a^{E}\left(\boldsymbol{v}-\widehat{\Pi}^{E} \boldsymbol{v}, \boldsymbol{v}-\widehat{\Pi}^{E} \boldsymbol{v}\right) \\
& \leq \max \left\{1, c_{1}\right\}\left(a^{E}\left(\widehat{\Pi}^{E} \boldsymbol{v}, \widehat{\Pi}^{E} \boldsymbol{v}\right)+a^{E}\left(\boldsymbol{v}-\widehat{\Pi}^{E} \boldsymbol{v}, \boldsymbol{v}-\widehat{\Pi}^{E} \boldsymbol{v}\right)\right) \\
& =\alpha^{*} a^{E}(\boldsymbol{v}, \boldsymbol{v}),
\end{aligned}
$$

and similarly, setting $\alpha_{*}:=\min \left\{1, c_{0}\right\}$ :

$$
\begin{aligned}
a_{h}^{E}(\boldsymbol{v}, \boldsymbol{v}) & \geq \min \left\{1, c_{0}\right\}\left(a^{E}\left(\widehat{\Pi}^{E} \boldsymbol{v}, \widehat{\Pi}^{E} \boldsymbol{v}\right)+a^{E}\left(\boldsymbol{v}-\widehat{\Pi}^{E} \boldsymbol{v}, \boldsymbol{v}-\widehat{\Pi}^{E} \boldsymbol{v}\right)\right) \\
& =\alpha_{*} a^{E}(\boldsymbol{v}, \boldsymbol{v}) .
\end{aligned}
$$


Note that (5.10) and (2.12), in particular, imply

$$
a_{h}(\boldsymbol{v}, \boldsymbol{v}) \geq \alpha_{*} \alpha\|\boldsymbol{v}\|_{H}^{2} \quad \forall \boldsymbol{v} \in V_{h} .
$$

Moreover, introducing the discrete kernel

$$
\mathcal{K}_{h}:=\left\{\boldsymbol{v}_{h} \in V_{h} \text { s. t. } b\left(\boldsymbol{v}_{h}, q_{h}\right)=0 \forall q_{h} \in Q_{h}\right\} \equiv\left\{\boldsymbol{v}_{h} \in V_{h} \text { s.t. } \operatorname{div} \boldsymbol{v}=0\right\},
$$

we easily see from the definition of $V_{h}$ and $Q_{h}$ (see (3.4)-(3.5)) that

$$
\mathcal{K}_{h} \subset \mathcal{K},
$$

implying in particular that

$$
a_{h}(\boldsymbol{v}, \boldsymbol{v}) \geq \alpha_{*} \alpha_{0}\|\boldsymbol{v}\|_{V}^{2} \quad \forall \boldsymbol{v} \in \mathcal{K}_{h}
$$

Finally, we point out that the symmetry of $a_{h}$ together with (5.10) easily gives

$$
\begin{aligned}
a_{h}^{E}(\boldsymbol{u}, \boldsymbol{v}) & \leq\left(a_{h}^{E}(\boldsymbol{u}, \boldsymbol{u})\right)^{1 / 2}\left(a_{h}^{E}(\boldsymbol{v}, \boldsymbol{v})^{1 / 2} \leq \alpha^{*}\left(a^{E}(\boldsymbol{u}, \boldsymbol{u})\right)^{1 / 2}\left(a^{E}(\boldsymbol{v}, \boldsymbol{v})\right)^{1 / 2}\right. \\
& \leq \alpha^{*}\|a\|\|\boldsymbol{u}\|_{0, E}\|\boldsymbol{v}\|_{0, E} .
\end{aligned}
$$

Remark 5.3. The form (5.6) for $a_{h}$ is, in a sense, the trade mark of Virtual Element Methods (see for instance [4]), although its structure was already present in earlier works dealing with reduced integration for nodal quadrilateral elements (see e.g., $[23,24]$ and the references therein). We could say that the part

$$
a^{E}(\widehat{\Pi} \boldsymbol{u}, \widehat{\Pi} \boldsymbol{v})
$$

is the consistency part, that ensures (among other things) that the final method will satisfy the patch test (see always [4]). On the other hand, the second part

$$
\left\|\mathbb{K}^{-1}\right\| \mathcal{S}^{E}((I-\widehat{\Pi}) \boldsymbol{u},(I-\widehat{\Pi}) \boldsymbol{v})
$$

is in charge of stability: this is why it is made to vanish whenever $\boldsymbol{u}=\widehat{\Pi} \boldsymbol{u}$ or $\boldsymbol{v}=\widehat{\Pi} \boldsymbol{v}$ (so that it will not spoil the patch test), and only its order of magnitude and asymptotic behavior in $h$ matter.

At this point, we have just to check the discrete analogue of the inf-sup condition (2.13). We have the following theorem.

Theorem 5.4. Under the above assumptions, there exists a constant $\beta^{*}>0$, depending only on $\Omega$ and on the constant $C_{\mathcal{T}}$ in $\mathbf{H D}$, such that:

$$
\forall q^{*} \in Q_{h}, \exists \boldsymbol{w}_{h}^{*} \in V_{h} \text { such that } \operatorname{div} \boldsymbol{w}_{h}^{*}=q^{*} \text { and } \beta^{*}\left\|\boldsymbol{w}_{h}^{*}\right\|_{H} \leq\left\|q^{*}\right\|_{Q} .
$$

Proof. It is well known ( $c f$. e.g., Ladyzenskaya) that there exists a constant $\beta>0$, depending on $\Omega$, such that for every $q^{*} \in Q_{h} \subset Q$, there exists a $\boldsymbol{w}^{*} \in\left(H_{0}^{1}(\Omega)\right)^{2}$ such that

$$
\operatorname{div} \boldsymbol{w}^{*}=q^{*} \quad \text { and } \beta\left\|\boldsymbol{w}^{*}\right\|_{1, \Omega} \leq\left\|q^{*}\right\|_{Q} .
$$

Then we define its interpolant $\boldsymbol{w}_{I}^{*} \in V_{h}$ as in (4.4), and we have in particular, from (4.8) with $r=1$ and (5.23)

$$
\left\|\boldsymbol{w}_{I}^{*}\right\|_{H} \leq(1+C h)\left\|\boldsymbol{w}^{*}\right\|_{1, \Omega} \leq \frac{1+C h}{\beta}\left\|q^{*}\right\|_{Q}=: \frac{1}{\beta^{*}}\left\|q^{*}\right\|_{Q}
$$

Finally, we have from (4.6) and (5.23) that

$$
\operatorname{div} \boldsymbol{w}_{I}^{*}=P_{Q_{h}} \operatorname{div} \boldsymbol{w}^{*}=P_{Q_{h}} q^{*}=q^{*} .
$$


Corollary 5.5. The above result easily implies the classical inf-sup condition:

$$
\exists \widetilde{\beta}>0 \text { such that } \forall h, \forall \mathcal{T}_{h} \inf _{q \in Q_{h}} \sup _{\boldsymbol{v} \in V_{h}} \frac{(\operatorname{div} \boldsymbol{v}, q)}{\|q\|_{Q}\|\boldsymbol{v}\|_{V}} \geq \widetilde{\beta}>0 .
$$

Proof. Indeed, for every $q^{*} \in Q_{h}$, taking $\boldsymbol{w}_{h}^{*}$ as in (5.22), we have

$$
\left\|\boldsymbol{w}_{h}^{*}\right\|_{V}^{2}=\left\|\boldsymbol{w}_{h}^{*}\right\|_{H}^{2}+\left\|\operatorname{div} \boldsymbol{w}_{h}^{*}\right\|_{Q}^{2} \leq\left(\frac{1}{\left(\beta^{*}\right)^{2}}+1\right)\left\|q^{*}\right\|_{Q}^{2}
$$

so that

$$
\frac{\left(\operatorname{div} \boldsymbol{w}_{h}^{*}, q^{*}\right)}{\left\|\boldsymbol{w}_{h}^{*}\right\|_{V}\left\|q^{*}\right\|_{Q}}=\frac{\left\|q^{*}\right\|_{Q}}{\left\|\boldsymbol{w}_{h}^{*}\right\|_{V}} \geq \widetilde{\beta}
$$

with $\widetilde{\beta}=\beta^{*} / \sqrt{1+\left(\beta^{*}\right)^{2}}$, proving $(5.25)$.

\section{Convergence theorem}

We are now ready to introduce the discretized problem:

$$
\begin{cases}\text { Find }\left(\boldsymbol{u}_{h}, p_{h}\right) \text { in } V_{h} \times Q_{h} \text { such that } & \\ a_{h}\left(\boldsymbol{u}_{h}, \boldsymbol{v}\right)-b\left(\boldsymbol{v}, p_{h}\right)=0 & \forall \boldsymbol{v} \in V_{h}, \\ b\left(\boldsymbol{u}_{h}, q\right)=(f, q) & \forall q \in Q_{h} .\end{cases}
$$

We have the following theorem.

Theorem 6.1. Under the above assumptions, the discrete problem (6.1) has a unique solution $\left(\boldsymbol{u}_{h}, p_{h}\right)$. Moreover, for every approximation $\boldsymbol{u}_{\pi}$ of $\boldsymbol{u}$ that is piecewise in $\widehat{V}^{E}$ (as defined in (5.4)), we have

$$
\begin{gathered}
\left\|\boldsymbol{u}_{h}-\boldsymbol{u}\right\|_{H} \leq C_{1}\left(\left\|\boldsymbol{u}-\boldsymbol{u}_{I}\right\|_{H}+\left\|\boldsymbol{u}-\boldsymbol{u}_{\pi}\right\|_{H}\right), \\
\left\|p_{I}-p_{h}\right\|_{Q} \leq C_{2}\left(\left\|\boldsymbol{u}-\boldsymbol{u}_{h}\right\|_{H}+\left\|\boldsymbol{u}-\boldsymbol{u}_{\pi}\right\|_{H}\right),
\end{gathered}
$$

where $\boldsymbol{u}_{I}$ is defined in (4.4), $p_{I}$ is defined in (4.1), $C_{1}$ is a constant depending only on $\mathbb{K}, \alpha_{*}$, $\alpha^{*}$, and $C_{2}$ is a constant depending only on $\mathbb{K}, \alpha^{*}$ and $\beta^{*}$.

Proof. Existence and uniqueness of the solution of (6.1) follow immediately from (5.18) and (5.25). Then we note that the second equation of (6.1) gives $\operatorname{div} \boldsymbol{u}_{h}=P_{Q_{h}} f$, while from (4.6) we have $\operatorname{div} \boldsymbol{u}_{I}=P_{Q_{h}} \operatorname{div} \boldsymbol{u}=P_{Q_{h}} f$, so that, setting

$$
\boldsymbol{\delta}_{h}:=\boldsymbol{u}_{h}-\boldsymbol{u}_{I},
$$

we easily have first

$$
\operatorname{div} \boldsymbol{\delta}_{h}=0 .
$$

Hence, in particular,

$$
\boldsymbol{\delta}_{h} \in \mathcal{K}_{h} \subset \mathcal{K},
$$

and also

$$
\left\|\boldsymbol{\delta}_{h}\right\|_{V}=\left\|\boldsymbol{\delta}_{h}\right\|_{H} .
$$


Then we have:

$$
\begin{aligned}
& \alpha_{*} \alpha\left\|\boldsymbol{\delta}_{h}\right\|_{H}^{2} \leq(\text { use }(2.12) \text { and }(5.10)) \\
& \leq \alpha_{*} a\left(\boldsymbol{\delta}_{h}, \boldsymbol{\delta}_{h}\right) \leq a_{h}\left(\boldsymbol{\delta}_{h}, \boldsymbol{\delta}_{h}\right) \text { (use (6.4)) } \\
& =a_{h}\left(\boldsymbol{u}_{h}, \boldsymbol{\delta}_{h}\right)-a_{h}\left(\boldsymbol{u}_{I}, \boldsymbol{\delta}_{h}\right) \text { (use (6.1) with (5.1) and (6.5), and (5.7)) } \\
& =-\sum_{E} a_{h}^{E}\left(\boldsymbol{u}_{I}, \boldsymbol{\delta}_{h}\right)\left(\text { use } \pm \boldsymbol{u}_{\pi}\right) \\
& =-\sum_{E}^{E}\left(a_{h}^{E}\left(\boldsymbol{u}_{I}-\boldsymbol{u}_{\pi}, \boldsymbol{\delta}_{h}\right)+a_{h}^{E}\left(\boldsymbol{u}_{\pi}, \boldsymbol{\delta}_{h}\right)\right)(\text { use (5.9)) } \\
& =-\sum_{E}^{E}\left(a_{h}^{E}\left(\boldsymbol{u}_{I}-\boldsymbol{u}_{\pi}, \boldsymbol{\delta}_{h}\right)+a^{E}\left(\boldsymbol{u}_{\pi}, \boldsymbol{\delta}_{h}\right)\right)(\text { use } \pm \boldsymbol{u} \text { and (3.1)) } \\
& =-\sum_{E}^{E}\left(a_{h}^{E}\left(\boldsymbol{u}_{I}-\boldsymbol{u}_{\pi}, \boldsymbol{\delta}_{h}\right)+a^{E}\left(\boldsymbol{u}_{\pi}-\boldsymbol{u}, \boldsymbol{\delta}_{h}\right)\right)-a\left(\boldsymbol{u}, \boldsymbol{\delta}_{h}\right)(\text { use }(2.10) \text { with (6.5)) } \\
& =-\sum_{E}^{E}\left(a_{h}^{E}\left(\boldsymbol{u}_{I}-\boldsymbol{u}_{\pi}, \boldsymbol{\delta}_{h}\right)+a^{E}\left(\boldsymbol{u}_{\pi}-\boldsymbol{u}, \boldsymbol{\delta}_{h}\right)\right)
\end{aligned}
$$

Now we use the continuity of each $a^{E}$ and of each $a_{h}^{E}$ (that is, (5.19)) in (6.8) in order to obtain

$$
\left\|\boldsymbol{\delta}_{h}\right\|_{H}^{2} \leq C\left(\left\|\boldsymbol{u}_{I}-\boldsymbol{u}_{\pi}\right\|_{H}+\left\|\boldsymbol{u}-\boldsymbol{u}_{\pi}\right\|_{H}\right)\left\|\boldsymbol{\delta}_{h}\right\|_{H},
$$

for some constant $C$ depending only on $\Omega, \mathbb{K}, \alpha_{*}, \alpha^{*}$. Then (6.2) follows easily by the triangle inequality.

We turn next to the proof of (6.3). Taking $q^{*}:=p_{h}-p_{I}$, we can apply (5.22) and obtain a $\boldsymbol{w}_{h}^{*} \in V_{h}$ with $\operatorname{div} \boldsymbol{w}_{h}^{*}=q^{*}$ and

$$
\left\|\boldsymbol{w}_{h}^{*}\right\|_{H} \leq\left(1 / \beta^{*}\right)\left\|q^{*}\right\|_{Q}=\left(1 / \beta^{*}\right)\left\|p_{h}-p_{I}\right\|_{Q} .
$$

Hence, using the definition (4.1) of $p_{I}$ and the fact that $\operatorname{div} \boldsymbol{w}_{h}^{*} \in Q_{h}$, and then the first equations of (2.10) and (6.1), we deduce

$$
\left\|p_{h}-p_{I}\right\|_{Q}^{2}=\left\|q^{*}\right\|_{Q}^{2}=\left(p_{h}-p_{I}, \operatorname{div} \boldsymbol{w}_{h}^{*}\right)=\left(p_{h}-p, \operatorname{div} \boldsymbol{w}_{h}^{*}\right)=a_{h}\left(\boldsymbol{u}_{h}, \boldsymbol{w}_{h}^{*}\right)-a\left(\boldsymbol{u}, \boldsymbol{w}_{h}^{*}\right) .
$$

Splitting over the elements, and adding and subtracting $\boldsymbol{u}_{\pi}$, we have from (6.11)

$$
\begin{aligned}
\left\|p_{h}-p_{I}\right\|_{Q}^{2} & =\sum_{E \in \mathcal{T}_{h}}\left(a_{h}^{E}\left(\boldsymbol{u}_{h}-\boldsymbol{u}_{\pi}, \boldsymbol{w}_{h}^{*}\right)+a^{E}\left(\boldsymbol{u}_{\pi}-\boldsymbol{u}, \boldsymbol{w}_{h}^{*}\right)\right) \\
& \leq C\left(\left\|\boldsymbol{u}_{h}-\boldsymbol{u}_{\pi}\right\|_{H}+\left\|\boldsymbol{u}_{\pi}-\boldsymbol{u}\right\|_{H}\right)\left\|\boldsymbol{w}_{h}^{*}\right\|_{H} \\
& \leq C\left(\left\|\boldsymbol{u}_{h}-\boldsymbol{u}_{\pi}\right\|_{H}+\left\|\boldsymbol{u}_{\pi}-\boldsymbol{u}\right\|_{H}\right)\left\|p_{h}-p_{I}\right\|_{Q},
\end{aligned}
$$

where (6.10) was used in the last step. Hence (6.3) follows.

From the error estimates (6.2) and (6.3) we can then, with the usual techniques (see e.g., [12, 20]), derive estimates on the speed of convergence in terms of powers of $h$, the degree $k$ of accuracy, and the regularity of the solution. In particular we have

Corollary 6.2. Under the same assumptions of Theorem 6.1 we have

$$
\begin{gathered}
\left\|\boldsymbol{u}-\boldsymbol{u}_{h}\right\|_{H} \leq C h^{k+1}\|u\|_{k+1, \Omega} \quad\left\|\operatorname{div} \boldsymbol{u}-\operatorname{div} \boldsymbol{u}_{h}\right\|_{0, \Omega} \leq C h^{k}\|f\|_{k, \Omega}, \\
\left\|p_{I}-p_{h}\right\|_{Q} \leq C h^{k+1}\|\boldsymbol{u}\|_{k+1, \Omega} \quad\left\|p-p_{h}\right\|_{0, \Omega} \leq C h^{k}\left(\|p\|_{k, \Omega}+\|\boldsymbol{u}\|_{k, \Omega}\right) .
\end{gathered}
$$

Note that, in particular, the first estimate of (6.14) should be regarded as a superconvergence result (since the pressure is locally in $\mathbb{P}_{k-1}$ ), very much in the spirit of [21]. 
Remark 6.3. In our construction, we started from the degree $k$ of the normal component on each edge, and then we chose $k-1$ as the local degree of the divergence, of the curl, and of the elements in $Q_{h}$. This gave us spaces that are sort of BDM-like. Our choice was dictated by the effort to remain as simple as possible. Obviously we could have been slightly more general. One can rather easily see that taking four integers $k_{e}, k_{d}$, $k_{r}$, and $k_{q}$, we could define the local space of vectors as

$V_{h}^{E}:=\{\boldsymbol{v} \in H(\operatorname{div} ; E) \cap H(\operatorname{rot} ; E)$ such that

$$
\left.(\boldsymbol{v} \cdot \boldsymbol{n})_{\mid e} \in \mathbb{P}_{k_{e}}(e) \text { for all edge } e \text { of } E,(\operatorname{div} \boldsymbol{v})_{\mid E} \in \mathbb{P}_{k_{d}}(E) \text { and }(\operatorname{rot} \boldsymbol{v})_{\mid E} \in \mathbb{P}_{k_{r}}(E)\right\},
$$

and take as $Q_{h}$ the space of scalars locally in $\mathbb{P}_{k_{q}}$. Taking $k_{e}$ instead of $k$ in (3.8)-1, $k_{d}$ instead of $k-1$ in (3.8)-2, and finally $k_{r}$ instead of $k-1$ in (3.8)-3, we see that we could easily carry out all our construction of Section 3. Needless to say, the local space for vectors will now contain all the polynomials of degree

$$
k=\min \left\{k_{e}, k_{d}+1, k_{r}+1\right\},
$$

so that, in general, $k_{d} \geq k_{e}-1$ and $k_{r} \geq k_{e}-1$ would be required in order to avoid a loss of accuracy in the approximation properties of our spaces. Moreover, in the study of stability, we would actually need $k_{q}=k_{d}$. Indeed, for $k_{q}<k_{d}$ we would lose the inclusion of kernels (5.17), and for $k_{q}>k_{d}$ we would lose the inf-sup condition (5.22). Hence our choice $\left(k_{q}=k_{d}=k_{e}-1\right.$ and $\left.k_{r}=k_{e}-1\right)$ can be seen as minimal and corresponds, as we said, to some sort of $B D M$-like elements. But we could have chosen

$$
k_{q}=k_{d}=k_{e} \quad k_{r}=k_{e}-1
$$

to get some $R T$-like (or rather $B D F M$-like) elements (see e.g., [14] or [11] for all this terminology). Note that with the choice (6.16) we could allow $k_{q}=k_{d}=k_{e}=0$ and $k_{r}=-1$ (with the usual convention that $\mathbb{P}_{-1} \equiv\{0\}$ ) that would mimic the lowest order Raviart-Thomas element.

Finally we point out that, instead of requiring the local vector space to contain all vector polynomials of a certain degree $\left(\right.$ say, $\left.\left(\mathbb{P}_{k}\right)^{2}\right)$, we could limit ourselves to the minimal requirement that the local vector space contains the spaces of the type $\mathbb{K} \operatorname{grad} q$ for all $q$, say, in $\in \mathbb{P}_{k+1}$ (as we used here in (5.4)). In this case we can change the requirement $\operatorname{rot} \boldsymbol{v} \in \mathbb{P}_{k_{r}}$ into $\operatorname{rot}\left(\mathbb{K}^{-1} \boldsymbol{v}\right)=0$, and get away, in general, with less degrees of freedom. All these choices, however, cannot yet be reasonably discussed, in the absence of suitable numerical experiments.

Remark 6.4. It is interesting to note that, since all our construction is done on the current element (and we don't use the reference element), on general quadrilaterals our Virtual Elements will not incur the troubles of classical BDM elements for "non affine quadrilaterals" (see [2]), and should therefore be compared to the ABF elements in [3].

Remark 6.5. It is clear that (as it happens for practically all $H$ (div)-conforming Finite Elements) a rotation of our vector fields of 90 degrees $\left(\left(u_{1}, u_{2}\right) \rightarrow\left(-u_{2}, u_{1}\right)\right)$ would generate $H$ (rot)-conforming vector fields.

Remark 6.6. The extension of the $H$ (div)-conforming elements introduced here to the three-dimensional case is essentially straightforward, giving rise to 3-dimensional face elements. However the extension to three dimensions of their $H$ (rot)-conforming version (in the spirit of the above remark) is less trivial. Mixed 3D elements, of both types, are the object of a paper in preparation [6].

Remark 6.7. The extension of the present methods to more general problems, including for instance variable permeability, the presence of lower order terms, and nonlinearities, is still to be done. Good suggestions for the treatment of a variable permeability can be obtained from previous works on Mimetic Finite Differences (as, for instance, [7]). For the treatment of lower order terms, a natural approach is to generalize the technique used in [1] for nodal Virtual Elements. 


\section{REFERENCES}

[1] B. Ahmad, A. Alsaedi, F. Brezzi, L.D. Marini and A. Russo, Equivalent projectors for virtual element methods, Comput. Math. Appl. 66 (2013) 376-391.

[2] D.N. Arnold, D. Boffi and R.S. Falk, Approximation by quadrilateral finite elements. Math. Comput. 71 (2002) 909-922.

[3] D.N. Arnold, D. Boffi and R.S. Falk, Quadrilateral H(div) finite elements. SIAM J. Numer. Anal. 42 (2005) $2429-2451$.

[4] L. Beirão da Veiga, F. Brezzi, A. Cangiani, L.D. Marini, G. Manzini and A. Russo, The basic principles of Virtual Elements Methods. Math. Models Methods Appl. Sci. 23 (2013) 199-214.

[5] L. Beirão da Veiga, F. Brezzi and L.D. Marini, Virtual Elements for linear elasticity problems. SIAM J. Num. Anal. 51 (2013) $794-812$.

[6] L. Beirão da Veiga, F. Brezzi, L.D. Marini and A. Russo, Mixed Virtual Element Methods in three dimensions. In preparation.

[7] L. Beirão da Veiga, K. Lipnikov and G. Manzini, Convergence analysis of the high-order mimetic finite difference method. Numer. Math. 113 (2009) 325-356.

[8] L. Beirão da Veiga, K. Lipnikov and G. Manzini, Arbitrary-Order Nodal Mimetic Discretizations of Elliptic Problems on Polygonal Meshes. SIAM J. Numer. Anal. 49 (2011) 1737-1760.

[9] L. Beirão da Veiga and G. Manzini, A higher-order formulation of the Mimetic Finite Difference Method SIAM J. Sci. Comput. 31 (2008) 732-760.

[10] P. Bochev and J.M. Hyman, Principle of mimetic discretizations of differential operators, Compatible discretizations. In vol. 142 of Proc. of IMA hot topics workshop on compatible discretizations. Edited by D. Arnold, P. Bochev, R. Lehoucq, R. Nicolaides and M. Shashkov. Springer-Verlag (2006).

[11] D. Boffi, F. Brezzi and M. Fortin, Mixed Finite Element Methods and Applications. Springer-Verlag, New York (2013).

[12] S.C. Brenner and R.L. Scott, The mathematical theory of finite element methods. In vol. 15 of Texts Appl. Math. SpringerVerlag, New York (2008).

[13] F. Brezzi, A. Buffa and K. Lipnikov, Mimetic finite differences for elliptic problems. ESAIM: M2AN 43 (2009) $277-295$.

[14] F. Brezzi and M. Fortin, Mixed and Hybrid Finite Element Methods. Springer-Verlag, New York (1991).

[15] F. Brezzi, K. Lipnikov and M. Shashkov, Convergence of mimetic finite difference method for diffusion problems on polyhedral meshes. SIAM J. Num. Anal. 43 (2005) 1872-1896.

[16] F. Brezzi, K. Lipnikov, M. Shashkov and V. Simoncini, A new discretization methodology for diffusion problems on generalized polyhedral meshes. Comput. Meth. Appl. Mech. Engrg. 196 (2007) 3682-3692.

[17] F. Brezzi, K. Lipnikov and V. Simoncini, A family of mimetic finite difference methods on polygonal and polyhedral meshes. Math. Models Methods Appl. Sci. 15 (2005) 533-1553.

[18] F. Brezzi and L.D. Marini, Virtual elements for plate bending problems. Comput. Meth. Appl. Mech. Engrg. 253 (2013) 155-462.

[19] A. Cangiani, G. Manzini and A. Russo, Convergence analysis of the mimetic finite difference method for elliptic problems. SIAM J. Numer. Anal. 47 (2009) 2612-2637.

[20] P.G. Ciarlet, The Finite Element Method for Elliptic Problems. North-Holland (1978).

[21] J. Douglas, Jr. and J.E. Roberts, Mixed finite element methods for second order elliptic problems. Math. Appl. Comput. 1 (1982) 91-103.

[22] J. Droniou, R. Eymard, T. Gallouët and R. Herbin, A unified approach to mimetic finite difference, hybrid finite volume and mixed finite volume methods. Math. Models Methods Appl. Sci. (M3AS) 20 (2010) 265-295.

[23] C.A. Felippa, Supernatural QUAD4: A template formulation Comput. Methods Appl. Mech. Engrg. 195 (2006) 5316-5342.

[24] T.-P. Fries and T. Belytschko, The extended/generalized finite element method: An overview of the method and its applications Int, J. Numer. Meth. Engng. 84 (2010) 253-304.

[25] A. Gain and G.H. Paulino, Phase-field based topology optimization with polygonal elements: a finite volume approach for the evolution equation. Struct. Multidiscip. Optim. (2012) 4632-7342.

[26] V. Gyrya and K. Lipnikov, High-order mimetic finite difference method for diffusion problems on polygonal meshes. J. Comput. Phys. 227 (2008) 8841-8854.

[27] J.M. Hyman and M. Shashkov, The orthogonal decomposition theorems for mimetic finite difference methods. SIAM J. Numer. Anal. 36 (1999) 788-818.

[28] Yu. Kuznetsov and S. Repin, New mixed finite element method on polygonal and polyhedral meshes. Russ. J. Numer. Anal. Math. Model. 18 (2003) 261-278.

[29] S. Rjasanow and S. Weißer, Higher order BEM-based FEM on polygonal meshes. SIAM J. Numer. Anal. 50 (2012) $2357-2378$.

[30] A. Tabarraei and N. Sukumar, Conforming polygonal finite elements. Int. J. Numer. Meth. Engrg. 61 (2004) $2045-2066$.

[31] A. Tabarraei and N. Sukumar, Extended finite element method on polygonal and quadtree meshes. Comput. Methods Appl. Mech. Engrg. 197 (2007) 425-438.

[32] C. Talischi, G.H. Paulino and C.H. Le, Honeycomb Wachspress finite elements for structural topology optimization. Struct. Multidiscip. Optim. 37 (2009) 569-583.

[33] E. Wachspress, A rational Finite Element Basis. Academic Press, New York (1975). 